\title{
Enhancing the stability of a fractional Chern insulator against competing phases
}

\author{
Adolfo G. Grushin, ${ }^{1,2}$ Titus Neupert, ${ }^{3}$ Claudio Chamon, ${ }^{2}$ and Christopher Mudry ${ }^{3}$ \\ ${ }^{1}$ Instituto de Ciencia de Materiales de Madrid, CSIC, Cantoblanco, E-28049 Madrid, Spain \\ ${ }^{2}$ Physics Department, Boston University, Boston, Massachusetts 02215, USA \\ ${ }^{3}$ Condensed Matter Theory Group, Paul Scherrer Institute, CH-5232 Villigen PSI, Switzerland
}

(Received 27 July 2012; published 21 November 2012)

\begin{abstract}
We construct a two-band lattice model whose bands can carry the Chern numbers $C=0, \pm 1, \pm 2$. By means of numerical exact diagonalization, we show that the most favorable situation that selects fractional Chern insulators (FCIs) is not necessarily the one that mimics Landau levels, namely a flat band with Chern number 1. First, we find that the gap, measured in units of the on-site electron-electron repulsion, can increase by almost two orders of magnitude when the bands are flat and carry a Chern number $C=2$ instead of $C=1$. Second, we show that giving a width to the bands can help to stabilize a FCI. Finally, we put forward a tool to characterize the real-space density profile of the ground state that is useful to distinguish FCI from other competing phases of matter supporting charge density waves or phase separation.
\end{abstract}

DOI: 10.1103/PhysRevB.86.205125

PACS number(s): 73.43.Cd

\section{INTRODUCTION}

A large effort in condensed-matter physics is dedicated to the classification of distinct phases of matter, exploring their properties, and establishing their robustness. Many phases of matter can be classified by the pattern in which a ground-state manifold breaks spontaneously and locally a symmetry of the underlying many-body Hamiltonian. The experimental discovery in Ref. 1 of the fractional quantum Hall effect (FQHE) in high-quality $\mathrm{GaAs}-\mathrm{Al}_{x} \mathrm{Ga}_{1-x} \mathrm{As}$ heterostructures subjected to very large magnetic field in 1982 was revolutionary in that the FQHE is associated to a family of phases of matter that are not characterized by some pattern of spontaneous symmetry breaking with a local order parameter, but instead by the notion of topological order. ${ }^{2,3}$ By analogy with spontaneous symmetry breaking and its connection to (Lie) group theory, a classification of topological order for many-body Hamiltonians has been undertaken. ${ }^{3}$

The study of all possible patterns of spontaneous symmetry breaking or topological order, although immensely useful, fails to address a very important question. What is the mechanism by which a given pattern associated to a phase is established? This is a quantitative question that requires deciding in the simplest case of two competing terms in a many-body Hamiltonian (free energy) which one is the dominant one. Answering this question, although essential from a practical point of view, can be very difficult. Detailed prescriptions of the Hamiltonian matter so that nonuniversality rules when calculating the location in the phase diagram of the boundaries separating phases of matter. In practice, a realistic answer to this question requires modern computing power if at all possible.

A problem related to the stability of phases is as follows. Suppose that we are given two physically different realizations of the same universal phase: Are the most favorable conditions for realizing the phase in one physical setting the same as in the other? More specifically, this paper analyzes the optimal situation for stabilizing a fractional Chern insulator (FCI). It also compares and contrasts it to the optimal situation for stabilizing a FQHE in a Landau level.
Fractional Chern insulators are strongly correlated phases of matter for interacting fermions and bosons that have been found in Bloch bands with vanishing or small bandwidth and nonvanishing Chern number. ${ }^{4-16}$ Fermionic FCIs share the universal properties of the FQHE that occurs upon partial filling of Landau levels. It was thus not surprising that the efforts to establish that FCIs can be realized in model Hamiltonians began by mimicking as closely as possible the conditions for obtaining the FQHE in Landau levels. Bloch bands were chosen so as to try to mirror the properties of the Landau levels, which are special because (I) they support a topological attribute called a Chern number that equals 1 , and (II) they are independent of their momentum quantum number; i.e., they can be thought of as flat bands. Bloch bands can be made to satisfy properties (I) and (II). ${ }^{4}$ However, even if the Bloch bands are made to satisfy conditions (I) and (II), it is not a priori obvious that energetics allow the formation of a fractional quantum Hall ground state. ${ }^{17-21}$ For example, the filling fraction $1 / 3$ is commensurate to the lattice; hence, one might expect that, instead of a fractional Chern state, interactions select a charge density wave as the ground state. Nevertheless, it has become apparent from exact diagonalization studies of FCIs that the universal properties of the FQHE can be stabilized by starting from filling partially a (sufficiently) flat band of a Chern band insulator for some short-range interaction.

That one can deviate slightly from condition (II) without destroying the FCI phase is expected, for the topological phase is gapped and therefore stable to small perturbations. In contrast, one cannot deviate continuously from condition (I), for the Chern number is an integer. The work presented below aims to analyze cases when departing from the conditions (I) and (II), associated to the FQHE in Landau bands, can lead to enhanced stability of the FCIs.

We present a lattice model for which the Chern number can take values $C=0, \pm 1, \pm 2$. We show that it is more difficult to stabilize a FCI with $C=1$ than a FCI with $C=2$ due to energetics for this model. We find that the gaps for a FCI at the filling fraction $v=1 / 5$ when $C=2$ are almost two orders of magnitude larger than those for the most stable fractional 
Chern state that we found for the filling fraction $v=1 / 3$ when $C=1$.

To establish that the state at $v=1 / 5$ is a FCI and not some competing state that breaks spontaneously and locally some space-group symmetry of the lattice, we present in this paper a useful tool to distinguish fractional Chern states from charge density wave (CDW) states even when the lattices studied are rather small. We employ this diagnostic to map out the phase diagram that should emerge in the thermodynamic limit as a function of the strength of interactions, and to identify the nature of the phases that compete with the $v=1 / 5 \mathrm{FCI}$ at $C=2$. In doing so, we substantiate our claim that a FCI can be stabilized by moving away from property (I) obeyed by the Landau levels.

We then proceed to study the effects of relaxing the flatness condition (II). We argue that relaxing condition (II) can help stabilize a FCI. We do this in two ways.

First, we appeal to a general argument that relies on a particle-hole transformation of any Hamiltonian $H$ for itinerant and interacting electrons with a topological flat band. By construction, if $H$ supports as a ground state a FCI at the filling fraction $v$, then the particle-hole transformed Hamiltonian $\widetilde{H}$ supports a FCI at the filling fraction $\widetilde{v}=$ $1-v$. We show that if the interaction is a two-body one with translational symmetry, then $\widetilde{H}$ acquires a one-body contribution through normal ordering. This simple observation has the following remarkable consequence. A FCI at the filling fraction $\widetilde{v}$ might exist even though $\widetilde{H}$ can be decomposed into a one-body term that generates a band width of the same order as the normal-ordered two-body interaction. Conversely, if this one-body term is switched off adiabatically, the ground state at the filling fraction $\widetilde{v}$ might undergo a phase transition to a phase that does not support a topological order. In fact, this is the explanation for the numerical observation that distinct $H$ at the filling fractions $v=1 / 3$ or $v=1 / 5$ support FCIs as the ground states, while the very same $H$ at the filling fractions $\widetilde{v}=2 / 3$ or $\widetilde{v}=4 / 5$ are not topologically ordered..$^{22-24}$

Second, in order to probe the phase diagram of the system as a function of two parameters, one of which controls the size of the bandwidth, we use another numerical tool to help trace out the phase boundaries of topological states using exact diagonalization studies of small lattices. We look at the ratio between the observed gap $\Delta$ and the spread in energy $\delta$ of those states that belong to the manifold of states that are to become degenerate in the thermodynamic limit. If $L$ is the characteristic linear size of the lattice, this ratio $\Delta / \delta \sim \exp (L / \xi)$ entails information on the correlation length $\xi$ of the system. The correlation length $\xi$ is a useful mean to measure the distance to the phase boundary. By combining the parametric dependence of $\Delta$ along contours at constant $\Delta / \delta$, we can estimate the region of stability of the FCI at $v=1 / 5$ and $C=2$ from exact diagonalization. We find that moving away from condition (II) that is obeyed by Landau levels, i.e., including a nonzero bandwidth, can (up to a limit) help increase (and not decrease) the range of phase stability.

\section{THE MODEL}

Consider a noninteracting tight-binding model for fermions on the two-dimensional square lattice $\Lambda$ made of $L_{1} \times L_{2}$ sites and spanned by the orthonormal primitive lattice vectors $\hat{\boldsymbol{e}}_{1}$ and $\hat{\boldsymbol{e}}_{2}$. We impose periodic boundary conditions. The fermions have two internal degrees of freedom per site, which we denote as a spin degree of freedom $s=\uparrow, \downarrow$. Each hopping process is associated with a spin flip. The resulting noninteracting Bloch Hamiltonian supports two bands and reads in momentum space

$$
\begin{aligned}
H_{0}:= & \sum_{\boldsymbol{k} \in \mathrm{BZ}} c_{\boldsymbol{k}}^{\dagger} \boldsymbol{B}_{\boldsymbol{k}} \cdot \boldsymbol{\sigma} c_{\boldsymbol{k}}, \\
B_{\boldsymbol{k} ; 1}+\mathrm{i} B_{\boldsymbol{k} ; 2}:= & t\left(\sin k_{1}+\mathrm{i} \sin k_{2}\right), \\
B_{\boldsymbol{k} ; 3}:= & h_{1} \cos k_{1}+h_{2} \cos k_{2}+h_{3} \\
& +h_{4}\left[\cos \left(k_{1}+k_{2}\right)+\cos \left(k_{1}-k_{2}\right)\right],
\end{aligned}
$$

where $c_{\boldsymbol{k}}^{\dagger} \equiv\left(c_{\boldsymbol{k}, \uparrow}^{\dagger}, c_{\boldsymbol{k}, \downarrow}^{\dagger}\right)$ and $c_{\boldsymbol{k}, s}^{\dagger}$ creates a fermion at momentum $\boldsymbol{k}$ in the Brillouin zone (BZ) with spin $s=\uparrow, \downarrow$, while $\sigma=$ $\left(\sigma_{1}, \sigma_{2}, \sigma_{3}\right)$ are the three Pauli matrices acting on spin space. The parameters $t$ and $h_{\mu}, \mu=1, \ldots, 4$, are real.

The role of the hopping parameters $t$ and $h_{\mu}, \mu=1, \ldots, 4$, can be illustrated by fixing $t>0$ and expanding the Hamiltonian around the four inversion-symmetric momenta in the $\mathrm{BZ} \boldsymbol{k}^{(i j)}=\pi(i, j), i, j=0,1$. To linear order in the deviation $\boldsymbol{p}^{(i j)}=\boldsymbol{k}-\boldsymbol{k}^{(i j)}, i, j=0,1$, from each of these four momenta, the Hamiltonian takes a Dirac form with masses given by

$$
m^{(i j)}=(-1)^{i} h_{1}+(-1)^{j} h_{2}+h_{3}+(-1)^{i+j} 2 h_{4} .
$$

The model thus allows to independently control the sign and magnitude of the mass at each of the four Dirac points via the parameters $h_{\mu}, \mu=1, \ldots, 4$. If all the Dirac points have a nonvanishing gap, the Chern number of each of the two bands is well defined. Each Dirac point contributes $\pm 1 / 2$ to the Chern number, with the sign depending on the chirality $\exp \left(\mathrm{i} k_{1}^{(i j)}+\mathrm{i} k_{2}^{(i j)}\right)$ of the kinetic part of the Dirac Hamiltonian and the sign of the mass gap. The total Chern number of the lower band is then given by

$$
C=\frac{1}{2} \sum_{i, j=0,1}(-1)^{i+j} \operatorname{sgn} m^{(i j)}
$$

and therefore can assume the values $C= \pm 2, \pm 1,0$ in our two-band model (see Fig. 1).

In what follows, we study this model in presence of densitydensity interactions between fermions on the same lattice site $(U)$ and between fermions on neighboring sites $(V)$

$$
H_{\mathrm{int}}:=\frac{U}{2} \sum_{\boldsymbol{r} \in \Lambda} \sum_{s \neq s^{\prime}} \rho_{\boldsymbol{r}, s} \rho_{\boldsymbol{r}, s^{\prime}}+\frac{V}{2} \sum_{\left(\boldsymbol{r}, \boldsymbol{r}^{\prime}\right)} \sum_{s, s^{\prime}} \rho_{\boldsymbol{r}, s} \rho_{\boldsymbol{r}^{\prime}, s^{\prime}}
$$

upon partial filling the lower band. Here, $(\cdot, \cdot)$ denotes nearestneighbor lattice sites and $\rho_{\boldsymbol{r}, s}:=c_{\boldsymbol{r}, s}^{\dagger} c_{\boldsymbol{r}, s}$ is the density of fermions with spin $s=\uparrow, \downarrow$ at site $r \in \Lambda$.

We are interested in the situation where the following hierarchy of energy scales applies

$$
W \ll U, \quad V \ll m,
$$

with $W$ and $m$ being the bandwidth and the band gap of the noninteracting band structure, respectively. 
(a)
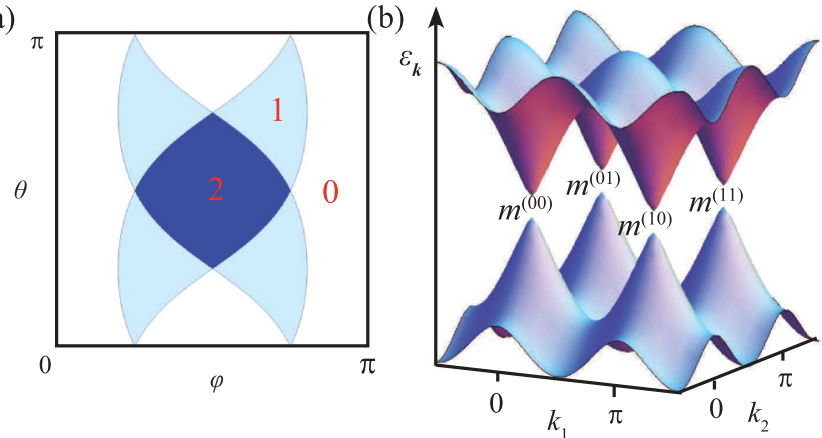

FIG. 1. (Color online) (a) Any one of the two Chern numbers of the two bands of the noninteracting Hamiltonian (2.1) depends on the direction of the four-component mass vector $h=\left(h_{\mu}\right)$. When restricted to the form $h=|h|(\cos \varphi, \sin \varphi \cos \theta, 0,(1 / 2) \sin \varphi \sin \theta)$, the Chern number phase diagram is obtained as shown (white, $|C|=0$; light, $|C|=1$; dark, $|C|=2$ ). (b) Band structure of the noninteracting Hamiltonian (2.1)) with mass terms $m^{(i j)}, i, j=0,1$, defined in Eq. (2.2a).

The fact that the band gap $m$ is the largest energy scale justifies considering the ideal limit,

$$
W / m \ll U / m, \quad V / m \rightarrow 0,
$$

in which the single-particle Hilbert space is projected onto the subspace spanned by the states in the lower band. Hence, we project the interaction (2.3) onto the Fock space built out of the single-particle subspace of the lower band. This projection gives

$$
\begin{aligned}
H_{\mathrm{int}}^{\mathrm{pro}}:= & \frac{-1}{L_{1} L_{2}} \sum_{\boldsymbol{k}, \boldsymbol{k}^{\prime}, \boldsymbol{q}} \gamma_{\boldsymbol{k}, \boldsymbol{k}^{\prime}, \boldsymbol{q}} \chi_{\boldsymbol{k}+\boldsymbol{q}}^{\dagger} \chi_{\boldsymbol{k}^{\prime}-\boldsymbol{q}}^{\dagger} \chi_{\boldsymbol{k}} \chi_{\boldsymbol{k}^{\prime}} \\
\gamma_{\boldsymbol{k}, \boldsymbol{k}^{\prime}, \boldsymbol{q}}:= & \sum_{s, s^{\prime}}\left[\frac{U}{2}\left(1-\delta_{s, s^{\prime}}\right)+V\left(\cos q_{1}+\cos q_{2}\right)\right] \\
& \times u_{\boldsymbol{k}+\boldsymbol{q}, s} u_{\boldsymbol{k}^{\prime}-\boldsymbol{q}, s^{\prime}} u_{\boldsymbol{k}, s}^{*} u_{\boldsymbol{k}^{\prime}, s^{\prime}}^{*}
\end{aligned}
$$

where $u_{k} \equiv\left(u_{k, s}\right)$ is the eigenvector of the lower band of the $2 \times 2$ Bloch Hamiltonian $\boldsymbol{B}_{\boldsymbol{k}} \cdot \boldsymbol{\sigma}$ in Eq. (2.1) at momentum $\boldsymbol{k}$, while $\chi_{\boldsymbol{k}}^{\dagger}$ is the second quantized operator that creates the corresponding state in the lower band.

The fact that $W$ is the smallest energy scale justifies considering the ideal limit in which the lower band is flat. This is achieved by defining the kinetic energy (see Ref. 4),

$$
H_{0}^{\text {flat }}:=\sum_{\boldsymbol{k} \in \mathrm{BZ}} c_{\boldsymbol{k}}^{\dagger} \frac{\boldsymbol{B}_{\boldsymbol{k}} \cdot \boldsymbol{\sigma}}{\left|\boldsymbol{B}_{\boldsymbol{k}}\right|} c_{k} .
$$

Here, dividing $\boldsymbol{B}_{\boldsymbol{k}} \cdot \boldsymbol{\sigma}$ by $\left|\boldsymbol{B}_{\boldsymbol{k}}\right|$ amounts to assigning to all Bloch states from the lower band the energy -1 and to all Bloch states from the upper band the energy +1 . This deformation of the Hamiltonian induces arbitrary-range hopping amplitudes in position space, but preserves its locality in the sense that these hopping amplitudes decay exponentially with distance. ${ }^{4}$ Interpolating between $H_{0}$ and $H_{0}^{\text {flat }}$ through the parametric dependence on $0 \leqslant \lambda \leqslant 1$ of

$$
H_{0}(\lambda):=(1-\lambda) H_{0}^{\text {flat }}+\lambda H_{0}
$$

makes it possible to choose the bandwidth at will.
We begin with the study of the case $\lambda=0$, using the Hamiltonian

$$
H(\lambda):=H_{0}(\lambda)+H_{\text {int }}^{\text {pro }}
$$

by carrying out exact diagonalization studies of small systems in Secs. III and IV. In Sec. V we study how a finite bandwidth (obtained by varying $\lambda$ ) affects the fractional Chern states.

\section{III. $C=1$ AND $C=2$ FRACTIONAL CHERN STATES}

The topological properties of the anticipated FCIs are believed to be described by a hierarchical Chern-Simons theory ${ }^{25-29}$ with the action

$$
S_{\mathrm{CS}}:=\frac{\varepsilon_{\mu \nu \lambda}}{4 \pi} \int \mathrm{d}^{2} r \mathrm{~d} t\left(-K_{i j} a_{i}^{\mu} \partial^{\nu} a_{j}^{\lambda}+2 e Q_{i} a_{i}^{\mu} \partial^{\nu} A^{\lambda}\right)
$$

in the continuum limit. Here $a_{i}^{1}, a_{i}^{2}$, and $a_{i}^{0}$ are the two spatial components and the temporal component of $i=1, \ldots, N_{\mathrm{f}}$ flavors of hydrodynamical gauge fields. The electromagnetic gauge field is denoted by $A^{\mu}, \mu=0,1,2$. The $N_{\mathrm{f}} \times N_{\mathrm{f}}$ matrix $K$ is symmetric with integer entries. The $N_{\mathrm{f}}$-component vector $Q=\left(Q_{i}\right)$ is the charge vector and it has integer entries. The summation convention over the repeated flavor and Greek indices is implied.

The topological ground-state degeneracy on the torus and the Hall conductivity are given by $\operatorname{det} K$ and

$$
\sigma_{\mathrm{H}}=\frac{e^{2}}{h} Q_{i} K_{i j}^{-1} Q_{j},
$$

respectively. On the other hand, we may naively expect the Hall conductivity of the fractional Chern states to be

$$
\sigma_{\mathrm{H}}=\frac{e^{2}}{h} C v
$$

where $v$ is the filling fraction of the lower band. When the band is fully filled $(v=1)$, indeed the expectation is fulfilled. That this formula holds for generic filling is always permitted but it is not guaranteed. When the Berry curvature is not constant in the BZ, $\sigma_{\mathrm{H}}$ does not need to be tied to $v$ anymore..$^{30,31}$

It is known that topologically degenerate ground states on the torus can be transferred into one another by adiabatically inserting flux through the torus. ${ }^{32}$ After an insertion of an integer number of flux quanta equal to the degeneracy, one must recover the same state. For a many-body state $|\Psi\rangle$ with $N$ particles inserting a flux $2 \pi \gamma_{i}$ in the $\hat{\boldsymbol{e}}_{i}$ direction is equivalent to imposing the twisted boundary conditions

$$
\begin{aligned}
& \left\langle\boldsymbol{r}_{1}, \ldots, \boldsymbol{r}_{j}+L_{i} \hat{\boldsymbol{e}}_{i}, \ldots, \boldsymbol{r}_{N} \mid \Psi\right\rangle \\
& \quad=e^{\mathrm{i} 2 \pi \gamma_{i}}\left\langle\boldsymbol{r}_{1}, \ldots, \boldsymbol{r}_{j}, \ldots, \boldsymbol{r}_{N} \mid \Psi\right\rangle, \quad j=1, \ldots, N .
\end{aligned}
$$

With this background to FCIs in mind, we proceed to discuss model (2.1). The principal advantage of this model is that it makes it possible to change the Chern number of the band from $C=-2$ to 2 , in steps of 1 . We first focus on the case $C=1$, which can be realized by choosing $h_{1}=h_{2}=-h_{3}=-t$ and $h_{4}=0$ and was previously studied in Ref. 10 . In this case, the model hosts a $1 / 3$ fractional Chern insulator state which we here include for completeness.

Figure 2(a) shows the low-energy portion of the manybody spectrum and the expected threefold quasidegenerate fractional Chern ground state when $U=t$ and $V=0$. The 
(a)

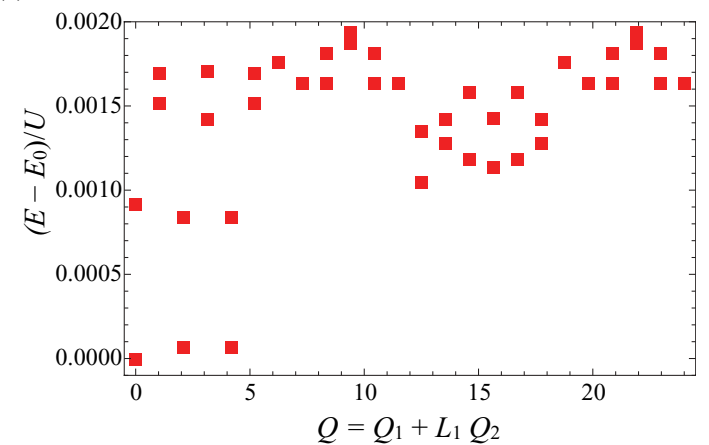

(b)

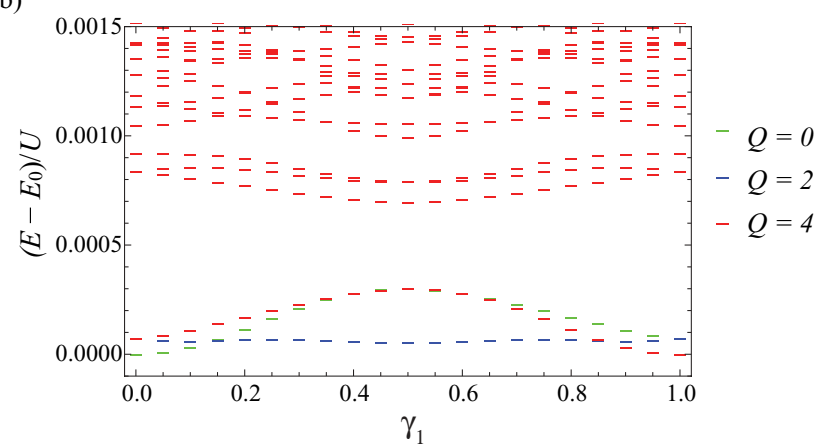

FIG. 2. (Color online) (a) Energy eigenvalues of Hamiltonian (2.8) at $\lambda=0$ measured relative to the ground-state energy at the filling fraction $v=1 / 3$ for $L=6 \times 4$ and $N=8$ particles as a function of the total momentum $\boldsymbol{Q}$. The noninteracting parameters of Hamiltonian (2.8) are $h_{4}=0$ and $h_{1}=h_{2}=-h_{3}=-t$, corresponding to a noninteracting band with $C=1$. The interacting parameters of Hamiltonian (2.8) are $U=t$ and $V=0$. A threefold quasidegenerate ground state is observed in agreement with Ref. 10. (b) Spectral flow induced by a flux insertion in the $\gamma_{1}$ direction for $N=8$ particles. The three lowest-lying states do not mix with the would-be continuum of excited states and return to the same configuration after three flux quanta have been inserted (only the insertion of one flux quantum is shown). For comparison with Fig. 3, the FCI at $v=1 / 3$ has disappeared when $V=t$.

spectrum is plotted as a function of the total center-of-mass momentum $\boldsymbol{Q}$. This is a good quantum number because the many-body Hamiltonian shares the space-group symmetry of the square lattice. The fact that the states fall in the sectors with center-of-mass momenta $\left(Q_{1}, Q_{2}\right)_{6 \times 4}=$ $\{(0,0),(2,0),(4,0)\}$ agrees with the counting rule introduced in Ref. 7. The threefold quasidegeneracy is consistent with a Chern-Simons theory (3.1) for a single species of gauge fields and $K=3, Q=1$.

Figure 2(b) shows the evolution of the spectrum when flux is adiabatically inserted in the $\hat{\boldsymbol{e}}_{1}$ direction for a $6 \times 4$ site lattice with $U=t, V=0$. One observes that the quasidegenerate groundstate manifold indeed evolves independently from the excited states. This observation is again consistent with a $v=$ $1 / 3$ fractional Chern state. Moreover, we checked that this state survives when $h_{4} \neq 0$ as long as the Chern number remains $C=1$.

With the help of Eq. (2.2b) one verifies that for sufficiently large $h_{4}>0$, the Chern number becomes $C=2$. In fact, the simplest set of parameters that hosts a Bloch band with $C=2$ is $h_{1}=h_{2}=h_{3}=0$ and $h_{4} \neq 0$. For this set of the parameters,

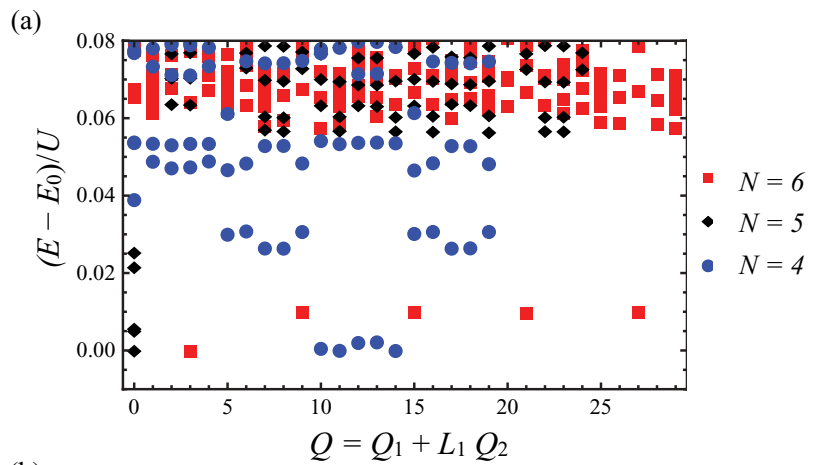

(b)

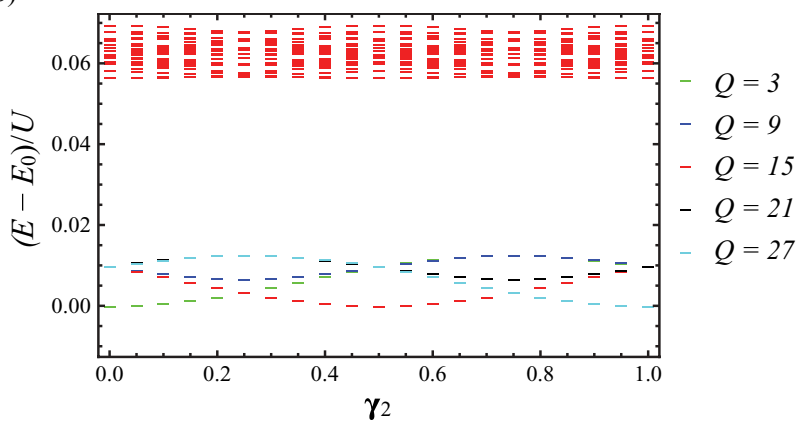

FIG. 3. (Color online) (a) Energy eigenvalues of Hamiltonian (2.8) at $\lambda=0$ measured relative to the ground state energy at the filling fraction $v=1 / 5$ for three different system sizes corresponding to $N=6,5$, and 4 particles (green diamonds, red squares, and black circles, respectively). The noninteracting parameters of Hamiltonian (2.8) are $h_{4}=0.7 t$ and $h_{i}=0$ for $i=1,2,3$ corresponding to $C=2$. The interacting parameters of Hamiltonian (2.8) are $U=V=t$. All three systems show a fivefold quasidegenerate ground state (see text for details). (b) Spectral flow induced by a flux insertion in the $\gamma_{2}$ direction for $N=6$ particles. The five lowest-lying states do not mix with the would-be continuum of excited states and return to the same configuration after five flux quanta have been inserted (only the insertion of one flux quantum is shown).

model (2.1) can be reinterpreted as a layered model where two square lattices are superimposed on each other without any hopping term that connects them. We are going to argue that when $h_{1}=h_{2}=h_{3}=0, h_{4}=0.7 t$, and $U=V=t$, the many-body Hamiltonian $H(\lambda=0)$ defined in Eq. (2.8) realizes a FCI at $v=1 / 5$ filling.

First, we show in Fig. 3(a) that for system sizes $6 \times 5$, $5 \times 5$, and $5 \times 4$ with $N=L_{1} L_{2} / 5$ particles, the many-body eigenvalues possess a fivefold degenerate ground state. The total momentum sectors $\boldsymbol{Q}$ at which these states should appear for a fractional Chern state can be calculated with a generalization of the counting rule of Ref. 7. For a $L_{1} \times L_{2}$ lattice with $N$ particles where we choose $L_{1}$ to be a multiple of five, the momentum sectors are given by

$$
\left(\begin{array}{l}
Q_{1} \\
Q_{2}
\end{array}\right)=\left(\begin{array}{c}
{\left[N\left(L_{1}-5\right) / 2+m N\right] \bmod L_{1}} \\
N\left(L_{2}-1\right) / 2 \bmod L_{2}
\end{array}\right),
$$

with $m=0,1,2,3,4$. If $L_{2}$ is a multiple of five the same rule applies upon interchanging $Q_{1}$ for $Q_{2}$. For the square lattices made of $6 \times 5,5 \times 5$, and $5 \times 4$ sites, the momentum sectors 
should fall at

$$
\begin{aligned}
& \left(\begin{array}{l}
Q_{1} \\
Q_{2}
\end{array}\right)_{6 \times 5}=\left(\begin{array}{l}
3 \\
0
\end{array}\right), \quad\left(\begin{array}{l}
3 \\
1
\end{array}\right), \quad\left(\begin{array}{l}
3 \\
2
\end{array}\right), \quad\left(\begin{array}{l}
3 \\
3
\end{array}\right), \quad\left(\begin{array}{l}
3 \\
4
\end{array}\right), \\
& \left(\begin{array}{l}
Q_{1} \\
Q_{2}
\end{array}\right)_{5 \times 5}=\left(\begin{array}{l}
0 \\
0
\end{array}\right), \quad\left(\begin{array}{l}
0 \\
0
\end{array}\right), \quad\left(\begin{array}{l}
0 \\
0
\end{array}\right),\left(\begin{array}{l}
0 \\
0
\end{array}\right),\left(\begin{array}{l}
0 \\
0
\end{array}\right), \\
& \left(\begin{array}{l}
Q_{1} \\
Q_{2}
\end{array}\right)_{5 \times 4}=\left(\begin{array}{l}
0 \\
2
\end{array}\right),\left(\begin{array}{l}
1 \\
2
\end{array}\right),\left(\begin{array}{l}
2 \\
2
\end{array}\right), \quad\left(\begin{array}{l}
3 \\
2
\end{array}\right),\left(\begin{array}{l}
4 \\
2
\end{array}\right),
\end{aligned}
$$

respectively. This is exactly what we find [see Fig. 3(a)].

Second, Fig. 3(b) shows the flux insertion in the $\hat{\boldsymbol{e}}_{2}$ direction for lattice size $L=6 \times 5$. The five states corresponding to the ground-state manifold evolve independently from the continuum and they only recover their original position after five flux quanta have been inserted in the system.

Third, we compute the Hall conductivity of the five quasidegenerate ground states via their many-body Chern number. To this end, we apply the discretized version,

$$
\widetilde{\sigma}_{\mathrm{H}}:=\frac{e^{2}}{h} \frac{2 \pi}{L_{1} L_{2}} \sum_{k \in \mathrm{BZ}} F_{k} \bar{n}_{k},
$$

of the formula derived in Ref. 31 for the quantum Hall conductivity $\sigma_{\mathrm{H}}$ as befits a finite lattice. Here, the singleparticle Berry curvature $F_{\boldsymbol{k}}$ and the many-body occupation number $\bar{n}_{k}$ averaged over the five quasidegenerate ground states $\left|\Psi_{i}\right\rangle, i=1, \ldots, 5$, are given by

$$
F_{\boldsymbol{k}}:=\mathrm{i} \sum_{s} \partial_{k_{2}} u_{\boldsymbol{k}, s}^{*} \partial_{k_{1}} u_{\boldsymbol{k}, s}-(1 \leftrightarrow 2)
$$

and

$$
\bar{n}_{k}:=\frac{1}{5 L_{1} L_{2}} \sum_{i=1}^{5}\left\langle\Psi_{i}\left|\chi_{k}^{\dagger} \chi_{k}\right| \Psi_{i}\right\rangle,
$$

respectively. It is shown in Ref. 31 that $\widetilde{\sigma}_{\mathrm{H}}$ converges to the Hall conductivity $\sigma_{\mathrm{H}}$ averaged over the degenerate ground states in the thermodynamic limit, provided no spontaneous symmetry-breaking of translation invariance occurs. Observe that the accuracy of the quantization of $\widetilde{\sigma}_{\mathrm{H}}$ is limited by the finite size of the system, as the Berry curvature (3.6b) is only summed over $L_{1} \times L_{2}$ points in the BZ to replace an integral in the thermodynamic limit. We have evaluated Eq. (3.6) for the values $h_{1}=h_{2}=h_{3}=0, h_{4}=0.7 t$, and $U=V=t$ in the many-body Hamiltonian $H(\lambda=0)$ defined by Eq. (2.8) for the rectangular lattices made of $5 \times 5,5 \times 6,3 \times 10$, and $2 \times 15$ sites. We obtain for $\widetilde{\sigma}_{\mathrm{H}}$ in units of $e^{2} / h$ the values $0.391,0.401,0.400$, and 0.500 , respectively. Results with the same numerical accuracy are obtained for each ground state individually, i.e., without the averaging over all ground states in Eq. (3.6c). We conclude that Eq. (3.3) with $C=2$ and $v=1 / 5$ is captured by Eq. (3.6a) within $2 \%$ accuracy for the rectangular lattices with the aspect ratios $5 \times 5,5 \times 6,3 \times 10$. The value of $\widetilde{\sigma}_{\mathrm{H}}$ for the aspect ratio $2 \times 15$ deviates from $\sigma_{\mathrm{H}}=0.4 e^{2} / h$ by $25 \%$. However, a ladder with one leg obeying periodic boundary conditions is topologically equivalent to a ring, not to a torus, as is the case for the $5 \times 5,5 \times 6,3 \times 10$ lattices.
All three observations are consistent with a FCI described by the Chern-Simons theory (3.1) for two species of gauge fields and

$$
K=\left(\begin{array}{ll}
3 & 2 \\
2 & 3
\end{array}\right), \quad Q=\left(\begin{array}{l}
1 \\
1
\end{array}\right) .
$$

Note that a single species of gauge fields with $K=5$ would not explain the Hall conductivity $\sigma_{\mathrm{H}}=2 e^{2} /(5 h)$.

Although these three pieces of evidence already point towards a $1 / 5$ fractional Chern state, this state could still be a CDW. To show that this is not the case, we put forward in Sec. IV a different tool to distinguish liquid states such as the fractional Chern states from other competing orders, namely phases with broken translational invariance such as CDW or phase-separated phases.

\section{TOPOLOGICAL ORDER AND LOCAL SYMMETRY BREAKING}

Both topological order and long-range order with a local order parameter are emergent phenomena that occur in the thermodynamic limit for a given dimensionality of space. Most model Hamiltonians are not exactly solvable in the thermodynamic limit. Approximations such as variational methods can be used to access the thermodynamic limit, but they are uncontrolled. Exact diagonalization techniques are not biased, but they are limited to finite sizes. The extrapolation of exact finite-size spectra to the thermodynamic limit must be undertaken with great care. In particular, this extrapolation can involve subtle dimensional crossovers (e.g., the thin torus limit discussed below).

In view of the effects of finite-size corrections to the thermodynamic limit, it is essential to use complementary probes for topological order in exact diagonalization studies to argue convincingly that a candidate FCI has the featureless character that is demanded from a topological fluid and to map out its boundary in the phase diagram. The particle entanglement spectrum has been used in Ref. 10 to identify FCIs. We are going to give another criterion that distinguishes a fractional Chern phase from a competing phase that breaks spontaneously the space-group symmetry of the lattice. Examples of such competing phases are CDWs and macroscopic phase separation. Equipped with this diagnostic, we are going to map out the phase diagram as a function of the dimensionless parameter $-2<V / U<1$ of Hamiltonian (2.8) with a flat band, i.e., $\lambda=0$.

Let $O_{\boldsymbol{r}}$ be any local operator defined for any site $\boldsymbol{r}$ from the lattice $\Lambda$. We assume that the ground-state manifold of $H(\lambda=0)$ is $n$-dimensional and spanned by the quasidegenerate ground states $\left|\Psi_{1}\right\rangle, \ldots,\left|\Psi_{n}\right\rangle$. By assumption, $H(\lambda=0)$ shares the space-group symmetry of the lattice $\Lambda$. Hence, we can always choose $\left|\Psi_{i}\right\rangle$ to be a simultaneous eigenstate of the momentum operator with the center of mass $\boldsymbol{Q}_{i}$ where $i=1, \ldots, n$. We define the $n \times n$ Hermitian matrix $\mathcal{O}_{r}$ with elements

$$
\mathcal{O}_{r ; i j}:=\left\langle\Psi_{i}\left|O_{r}\right| \Psi_{j}\right\rangle,
$$

which amounts to restricting the operator $O_{r}$ to its action on the ground-state manifold. Let $v_{O ; r}^{(i)}$ be the $i$ th eigenstate of the matrix $\mathcal{O}_{\boldsymbol{r}}$. Its eigenvalue $\lambda_{O}^{(i)}$ is independent of the 
lattice site $\boldsymbol{r}$ as a consequence of translation symmetry of the Hamiltonian. ${ }^{33}$ If the space-group symmetry of the lattice $\Lambda$ and of Hamiltonian $H(\lambda=0)$ is not to be broken spontaneously by the ground-state manifold, then it is necessary for all eigenvalues $\lambda_{O}^{(1)}, \ldots, \lambda_{O}^{(n)}$ of $\mathcal{O}_{r}$ to be equal. The spread of the eigenvalues measures the degree by which the symmetry associated with the operator $O_{\boldsymbol{r}}$ is broken. If $\lambda_{O}^{\min }$ and $\lambda_{O}^{\max }$ are, respectively, the minimum and maximum eigenvalues, the quantity to monitor is the difference

$$
\delta \lambda_{O}:=\lambda_{O}^{\max }-\lambda_{O}^{\min } .
$$

We demand that the condition $\delta \lambda_{O} \rightarrow 0$ holds in a suitable thermodynamic limit for all local operators if topological order is to hold.

We now apply this analysis for the case when $O_{r}=\rho_{r}$, the local density operator being defined by

$$
\rho_{\boldsymbol{r}}:=\frac{1}{L_{1} L_{2}} \sum_{\boldsymbol{q}, \boldsymbol{k}} \sum_{s} e^{\mathrm{i} \boldsymbol{q} \cdot \boldsymbol{r}} c_{\boldsymbol{k}+\boldsymbol{q}, s}^{\dagger} c_{\boldsymbol{k}, s},
$$

and construct the matrix with elements

$$
\varrho_{\boldsymbol{r} ; i j}:=\left\langle\Psi_{i}\left|\rho_{\boldsymbol{r}}\right| \Psi_{j}\right\rangle .
$$

A set of $n$ maps of the local fermion density in the groundstate manifold is obtained as follows for some finite lattice $\Lambda$. We denote with $v_{\rho ; r_{0}}^{(i)}, i=1, \ldots, n$ the set of orthonormal eigenvectors of $\varrho_{\boldsymbol{r}_{0}}$ at some arbitrarily chosen site $\boldsymbol{r}_{0}$ and evaluate the $n$ real functions

$$
n_{\boldsymbol{r}}^{(i)}:=v_{\rho ; \boldsymbol{r}_{0}}^{(i) \dagger} \varrho_{\boldsymbol{r}} v_{\rho ; \boldsymbol{r}_{0}}^{(i)}, \quad i=1, \ldots, n .
$$

The functions $n_{\boldsymbol{r}}^{(i)}$ are density maps of the $n$ linearly independent combinations of the states $\left|\Psi_{1}\right\rangle, \ldots,\left|\Psi_{n}\right\rangle$ selected by the set of eigenvectors $v_{\rho ; \boldsymbol{r}_{0}}^{(i)}, i=1, \ldots, n$. These functions show the variation of the local fermion density in position space, i.e., the finite lattice $\Lambda$. The extrapolation of these density maps to the thermodynamic limit can be used to distinguish between a ground-state manifold that supports a CDW, a phase separation in position space, or is featureless as would be expected from a FCI. We will give two examples. Example 1 is the case of the crossover of a fractional Chern state from a featureless liquid to a CDW as the aspect ratio of the lattice is varied. Example 2 is the case of a phase-separated ground state obtained with attractive interactions between the single-particle states from the flat band.

Fractional quantum Hall states on the torus turn smoothly into a CDW state, if the ratio $\min \left(L_{1}, L_{2}\right) / \ell \lesssim 1 .{ }^{34-37}$ Here, $\ell$ is the magnetic length. The counterpart to this so-called thin-torus limit also exists for fractional Chern insulators. ${ }^{38}$ For concreteness, let us consider the $v=1 / 5$ fractional Chern state at Chern number 2. For the aspect ratio $L_{1} / L_{2}=1$, as is the case for a lattice of $5 \times 5$ sites with five particles, all five topological ground states have the same center-of-mass momentum $\boldsymbol{Q}=\mathbf{0}$. Therefore, $\varrho(\boldsymbol{r})$ is the unit matrix for all $\boldsymbol{r} \in \Lambda$ and all functions $n_{\boldsymbol{r}}^{(i)}, i=1, \ldots, 5$, are independent of $\boldsymbol{r}$. As a consequence, the fractional Chern state is indeed featureless in this isotropic case. Upon choosing the slightly anisotropic lattice $L_{1}=5, L_{2}=6$, with $N=6$ particles, the eigenvalues of $\varrho(\boldsymbol{r})$ lie between 0.994 and 1.007. This gives rise to a small density variation in position space of about
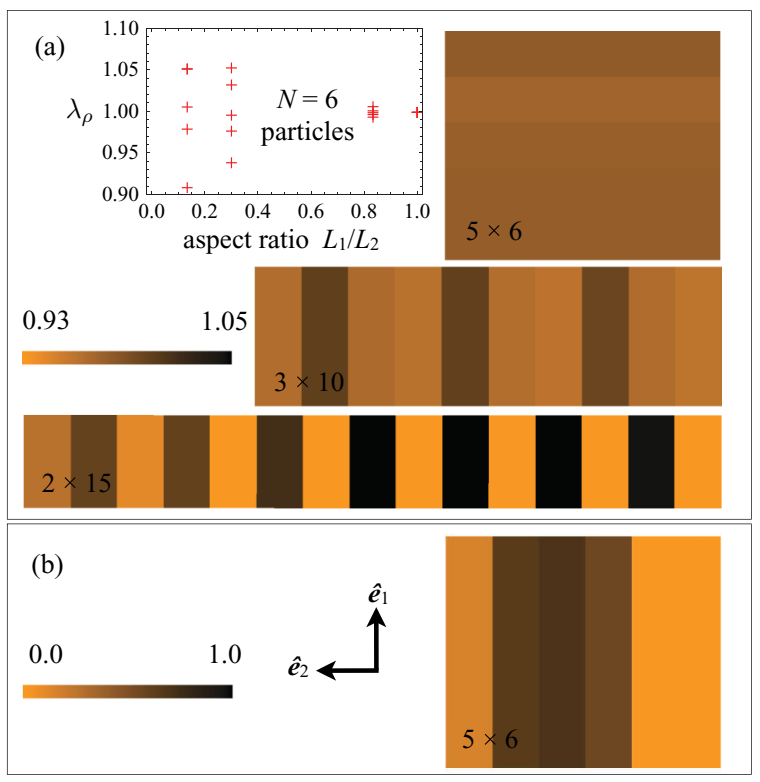

FIG. 4. (Color online) Density profile $n_{r}^{(i)}$ defined in Eq. (4.4) for one representative many-body ground state $\left|\Psi_{i}\right\rangle$ among the $n$ linearly independent quasidegenerate many-body ground states. Note the difference in the color scale between the plots in (a) and (b). (a) Case of the filling fraction $v=1 / 5$ when the representative ground states for $5 \times 6,3 \times 10$, and $2 \times 15$ lattices would turn into a fractional Chern insulator in the thermodynamic limit, by which the two linear dimensions of the lattice are much larger than the correlation length. In a finite lattice, a CDW profile becomes more pronounced when the aspect ratio mimics the thin-torus limit, by which the thermodynamic limit is taken with one of the two linear dimensions of the lattice comparable to or smaller than the correlation length. The inset shows the evolution of the eigenvalues $\lambda_{\rho}$ of the matrix $\varrho_{r ; i j}$ defined in Eq. (4.3b) as the aspect ratio of the lattice is changed. (b) Case of the filling fraction $v=1 / 5$ when the representative ground state for a $5 \times 6$ lattice would turn into a phase-separated ground state in the thermodynamic limit, by which the two linear dimensions of the lattice are much larger than the correlation length, as a result of attractive nearest-neighbor interactions.

$1 \%$ [see Fig. 4(a)]. Increasing further the anisotropy to $L_{1}=$ 2, $L_{2}=15$ with $N=6$ particles results in a spread of the eigenvalues of $\varrho(\boldsymbol{r})$ between 0.91 and 1.05 [see Fig. 4(a)]. In this case, the functions $n_{r}^{(i)}, i=1, \ldots, 5$, form the expected CDW pattern with pronounced density minima and maxima along the $\hat{\boldsymbol{e}}_{1}$ direction, as shown in Fig. 4(a).

We want to contrast these results with the density profile of the ground state that emerges when attractive instead of repulsive interactions are added to the noninteracting flat band model. We choose $U=t$ and vary $V$ from positive to negative values for a $L_{1}=5, L_{2}=6$ with $N=6$ particles. In doing so, we encounter two phase transitions towards different gapped ground states at about $V \sim 0$ and $V=-0.6 t$ [see Fig. 5(b)].

First, we obtain a state with macroscopic phase separation of the fermions and quasidegeneracy $L_{2}=6$. The strongly varying density profile in real space [see Fig. 4(b)] reveals that all particles cluster in a single stripe and the degeneracy emerges from shifting this stripe in the $\hat{\boldsymbol{e}}_{2}$ direction across the lattice. 
(a)

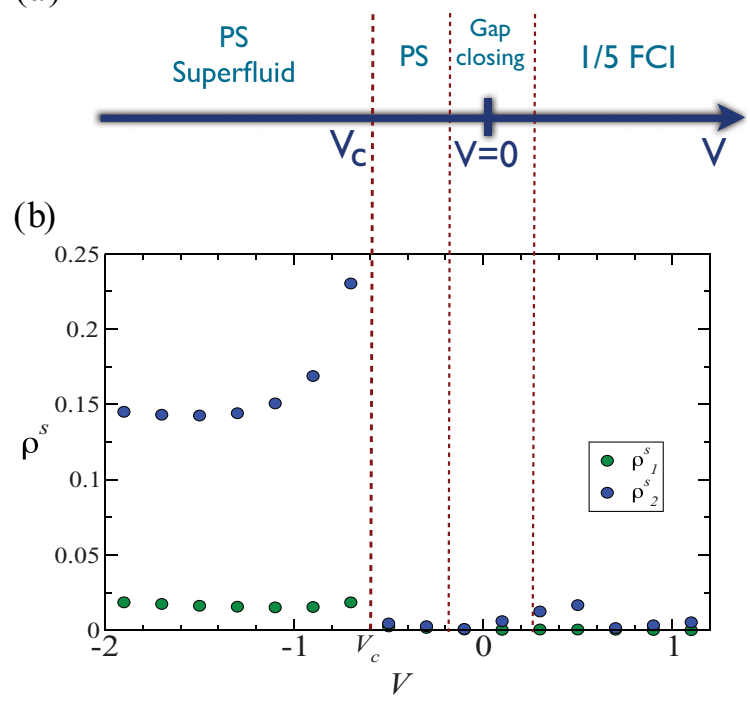

FIG. 5. (Color online) (a) Quantum phase diagram of Hamiltonian (2.8) at $\lambda=0$ for fixed noninteracting parameters corresponding to two flat bands with $C= \pm 2$ as a function of the nearestneighbor interaction strength $V$ for fixed on-site interaction strength $U=t$. The noninteracting parameters are $h_{4}=0.7 t$ and $h_{i}=0$ for $i=1,2,3$. There are three phases. For $V>0$ the $v=1 / 5$ fractional Chern insulator (FCI) is the most stable phase. For $V<0$ the system phase separates (PS) and turns superconducting below a critical $V_{\mathrm{c}} \sim-0.6 t$. (b) Superfluid phase stiffness as defined in Eq. (4.5) for the two orthogonal spanning directions of the lattice.

Second, a state with quasidegeneracy $30=L_{1} \times L_{2}$ is obtained with a density profile that shows a stripe similar to the first phase.

To characterize the physical difference between the two phases stabilized by an attractive $V$, we have computed the phase stiffness (see Refs. 39-41) of the ground state $|\Psi\rangle$ against twisting the boundary conditions away from periodic ones by a complex phase $\gamma_{i}, i=1,2$ according to Eq. (3.4). This stiffness equals the condensate fraction $\rho_{i}^{\mathrm{s}}$ of superfluid pairing in the state $|\Psi\rangle$ and it is given by

$$
\rho_{i}^{\mathrm{s}}=\left.\frac{\partial^{2} E_{0}(\boldsymbol{\gamma})}{\partial \gamma_{i}^{2}}\right|_{\gamma_{i}=0}, \quad i=1,2,
$$

where $E_{0} \equiv\langle\Psi|H(\lambda=0)| \Psi\rangle$ is the many-body ground-state energy. ${ }^{40}$ We find that in the phase with small negative $V$, there is no superfluid pairing, while the phase at large negative $V$ is a one-dimensional superconductor nucleated in the stripe of clustered particles. The phase stiffness is plotted for both directions in Fig. 5(b). The large increase of $\rho_{2}^{\mathrm{s}}$ when $V$ approaches $V_{\mathrm{c}} \sim-0.6 t$ from below suggests a phase transition. The direction for which the phase stiffness is the largest and diverging upon approaching the critical value $V_{\mathrm{c}} \sim-0.6 t$ from below is $\hat{\boldsymbol{e}}_{2}$. This is the direction of the stripe along which the electron density is uniform. Hence, it is plausible to interpret the phase $V<V_{\text {c }}$ as a stripelike superconducting phase.

\section{THE EFFECT OF BAND DISPERSION}

Having established the existence of fractional Chern ground states out of band insulators supporting the Chern numbers 1 and 2, we now study the effects on FCIs resulting from the spectrum of the noninteracting Hamiltonian not being flat. Bringing back the dispersion of the Bloch bands amounts to assigning a momentum-dependent energy penalty for occupying the single-particle states created (by $\chi_{k}^{\dagger}$ ) in the BZ. Increasing the bandwidth of the noninteracting Bloch bands to some critical value must result in a phase transition to a new correlated ground state, say a state that supports long-range order or a gapless featureless ground state such as a Fermi liquid. For a Laughlin state at filling $v$, lifting gradually the degeneracy of the Landau levels should gradually weaken the many-body gap, as we now argue. After projection into the lowest Landau level, the only remaining energy scale is the (screened) Coulomb interaction that delivers a many-body correlation length $\xi \sim \ell_{B}\left[\ell_{B}=\sqrt{\hbar c /(e B)}\right.$ the magnetic length]. Any small one-body perturbation that breaks Galilean invariance, an impurity potential or a periodic potential, brings about a characteristic length $\ell$. The effect of this one-body perturbation on the distance to a gap-closing phase transition should depend solely on the dimensionless ratio $\xi / \ell$, in which case a monotonically decreasing dependence is to be expected. Indeed, the Berry curvature of a Landau level is constant in momentum space. It does not favor any particular finite length scale. Thus, the distance to a gap-closing phase transition upon increasing $\ell$ should depend solely on the dimensionless ratio $\xi / \ell$. In contrast, a FCI with flat Bloch bands has a Berry curvature that must necessarily vary in momentum space. The Berry curvature might thus favor certain characteristic lengths. The distance to a gap-closing phase transition upon increasing $\ell$ should depend on more than one parameter, in which case nonmonotonic dependence on $\ell$ becomes a possibility.

We use two approaches to support this point. First, we give an analytical argument that applies to a generic lattice model for itinerant interacting fermions in the limit of a partially occupied flat band. We show how the addition of a fine-tuned band dispersion can be crucial to the selection of a FCI ground state. Second, we give supporting numerical evidences for a scenario by which switching on a finite bandwidth according to Eq. (2.7) can enhance the stability of a FCI for the specific model at hand.

For the analytical argument, consider an interacting Hamiltonian of the form

$$
H:=\sum_{\boldsymbol{k}_{1}, \boldsymbol{k}_{2}, \boldsymbol{k}_{3}, \boldsymbol{k}_{4}} V_{\boldsymbol{k}_{1} \boldsymbol{k}_{2} \boldsymbol{k}_{3} \boldsymbol{k}_{4}} \chi_{\boldsymbol{k}_{1}}^{\dagger} \chi_{\boldsymbol{k}_{2}}^{\dagger} \chi_{\boldsymbol{k}_{3}} \chi_{\boldsymbol{k}_{4}}
$$

that operates exclusively on the Fock space built out of the fermion creation operators $\chi_{k}^{\dagger}, \boldsymbol{k} \in \mathrm{BZ}$, of an isolated flat band. This model is generic for translational invariant density-density interactions which have matrix elements of the form

$$
V_{\boldsymbol{k}_{1} \boldsymbol{k}_{2} \boldsymbol{k}_{3} \boldsymbol{k}_{4}}=v_{\boldsymbol{k}_{1}-\boldsymbol{k}_{3}}\left\langle\chi_{\boldsymbol{k}_{1}} \mid \chi_{\boldsymbol{k}_{3}}\right\rangle\left\langle\chi_{\boldsymbol{k}_{2}} \mid \chi_{\boldsymbol{k}_{4}}\right\rangle \delta_{\boldsymbol{k}_{1}+\boldsymbol{k}_{2}, \boldsymbol{k}_{3}+\boldsymbol{k}_{4}}
$$

in the projected (flat) band, where $\left\langle\chi_{\boldsymbol{k}} \mid \chi_{\boldsymbol{k}^{\prime}}\right\rangle$ denotes the overlap between a pair of normalized single-particle Bloch states at 
$\boldsymbol{k}, \boldsymbol{k}^{\prime} \in \mathrm{BZ}$ and $v_{\boldsymbol{k}}$ is the Fourier component in the BZ of the unprojected and translational invariant two-body interaction.

Suppose that the ground state of $H$ at filling $v$ is a fractional Chern state [for instance, this is the case for the Hamiltonian (2.5) with $v=1 / 5]$. We apply a particle-hole transformation in this isolated band. This amounts to the transformation $\chi_{k}^{\dagger} \rightarrow \chi_{-k}, \chi_{k} \rightarrow \chi_{-k}^{\dagger}, k \in \mathrm{BZ}$. We obtain the transformed Hamiltonian

$$
\begin{aligned}
\tilde{H}= & \sum_{\boldsymbol{k}_{1}, \boldsymbol{k}_{2}, \boldsymbol{k}_{3}, \boldsymbol{k}_{4}} V_{-\boldsymbol{k}_{1},-\boldsymbol{k}_{2},-\boldsymbol{k}_{3},-\boldsymbol{k}_{4}} \chi_{\boldsymbol{k}_{3}}^{\dagger} \chi_{\boldsymbol{k}_{4}}^{\dagger} \chi_{\boldsymbol{k}_{1}} \chi_{\boldsymbol{k}_{2}} \\
& -\sum_{\boldsymbol{k}} \varepsilon_{-\boldsymbol{k}} \chi_{\boldsymbol{k}}^{\dagger} \chi_{\boldsymbol{k}}+\text { constant }
\end{aligned}
$$

in a normal-ordered form, where

$$
\begin{aligned}
\varepsilon_{\boldsymbol{k}}: & =\sum_{\boldsymbol{k}^{\prime}}\left(V_{\boldsymbol{k} \boldsymbol{k}^{\prime} \boldsymbol{k}^{\prime} \boldsymbol{k}}+V_{\boldsymbol{k}^{\prime} \boldsymbol{k} \boldsymbol{k} \boldsymbol{k}^{\prime}}\right) \\
& =\sum_{\boldsymbol{k}^{\prime}}\left(v_{\boldsymbol{k}-\boldsymbol{k}^{\prime}}+v_{\boldsymbol{k}^{\prime}-\boldsymbol{k}}\right)\left|\left\langle\chi_{\boldsymbol{k}} \mid \chi_{\boldsymbol{k}^{\prime}}\right\rangle\right|^{2} .
\end{aligned}
$$

We then conclude that, by construction, $\widetilde{H}$ supports a fractional Chern state (of holes) as its ground state at filling $\widetilde{v}=1-v$ [for instance, $\widetilde{H}$ when derived from Hamiltonian (2.5) at $v=$ $1 / 5$ supports a fractional Chern state (of holes) as its ground state at the filling $\widetilde{v}=4 / 5]$.

This fractional Chern state of holes at the filling fraction $\widetilde{v}=$ $1-v$ is stabilized in the presence of a one-body dispersion that is of the same order as the interaction itself and given by $\varepsilon_{\boldsymbol{k}}$. This one-body term can be interpreted as an optimal choice of the band dispersion that delivers as the ground state the fractional Chern state at the filling fraction $\widetilde{v}=1-v$. This one-body term is a trivial constant if and only if all overlaps between normalized Bloch states are functions of $\left(\boldsymbol{k}-\boldsymbol{k}^{\prime}\right)$ only. Conversely, if overlaps between normalized Bloch states also vary as a function of $\left(\boldsymbol{k}+\boldsymbol{k}^{\prime}\right)$, then the particle-hole transformed Hamiltonian $\widetilde{H}$ acquires a genuine one-body dispersion. Turning off adiabatically this genuine dispersion can induce a phase transition to a ground state that does not display a fractional (hole) state at the filling fraction $\widetilde{v}$. For instance, at the filling fraction $\widetilde{v}=4 / 5$, the Hamiltonians studied numerically in Refs. 14 and 15 can be interpreted as the Hamiltonian obtained from $\widetilde{H}$ upon subtracting the one-body term $\varepsilon_{k}$. These Hamiltonians at this filling fraction do not support a fractional Chern ground state, although $\widetilde{H}$ does.

We close this discussion by observing that the one-body dispersion (5.2b) induced by a particle-hole transformation has an elegant geometric interpretation that makes it possible to characterize FCIs though a local quantum metric tensor. The qualifier quantum originates from the fact that this local metric tensor is related to the overlaps of normalized Bloch states. From this geometrical point of view, we are going to show that the FQHE can be thought of as a FCI with a locally flat quantum metric tensor. To carry out this program, we observe that the one-body dispersion (5.2b) depends functionally on any one of the quantum distances (metrics)

$$
d_{k, k^{\prime}}(\kappa):=\sqrt{1-\left|\left\langle\chi_{k} \mid \chi_{k^{\prime}}\right\rangle\right|^{\kappa}},
$$

labeled by the real-valued parameter $\kappa \geqslant 1$ between the normalized single-particle Bloch states at $\boldsymbol{k}, \boldsymbol{k}^{\prime} \in \mathrm{BZ}$. With this definition, $d_{\boldsymbol{k}, \boldsymbol{k}^{\prime}}(\kappa)$ does indeed satisfy $d_{\boldsymbol{k}, \boldsymbol{k}^{\prime}}(\kappa)=0$ if and only if $\boldsymbol{k}=\boldsymbol{k}^{\prime}, d_{\boldsymbol{k}, \boldsymbol{k}^{\prime}}(\kappa)=d_{\boldsymbol{k}^{\prime}, \boldsymbol{k}}$, and the triangle inequality $d_{\boldsymbol{k}, \boldsymbol{k}^{\prime}}(\kappa) \leqslant d_{\boldsymbol{k}, \boldsymbol{k}^{\prime \prime}}+d_{\boldsymbol{k}^{\prime \prime}, \boldsymbol{k}^{\prime}}$ for any triplet of momenta from the BZ. Now the overlap between any pair of normalized Bloch state can be expressed in terms of any one of these metrics,

$$
\varepsilon_{\boldsymbol{k}}=\sum_{\boldsymbol{k}^{\prime}}\left(v_{\boldsymbol{k}-\boldsymbol{k}^{\prime}}+v_{\boldsymbol{k}^{\prime}-\boldsymbol{k}}\right)\left[1-d_{\boldsymbol{k}, \boldsymbol{k}^{\prime}}^{2}(\kappa)\right]^{2 / \kappa} .
$$

The so-called Fubini-Study metric, ${ }^{42}$ defined by selecting $\kappa=$ 1 in Eqs. (5.3a) and (5.3b), plays a special role, for it also enters in the algebra of projected density operators as shown by Roy in Ref. 43. For this reason, we select the Fubini-Study metric and drop the reference to $\kappa=1$ from now on. The usefulness of Eq. (5.3b) is rooted in the observation that, in the thermodynamic limit, if we introduce the local (Fubini-Study) metric tensor $g_{\mu \nu}(\boldsymbol{k})$ through the line integral along the paths $\gamma_{1,2}$ connecting the pair $\boldsymbol{k}_{1}$ and $\boldsymbol{k}_{2}$

$$
d\left(\boldsymbol{k}_{1}, \boldsymbol{k}_{2}\right)=\inf _{\gamma_{1,2}} \int_{\gamma_{1,2}} \mathrm{~d} \ell \sqrt{g_{\mu \nu}(\boldsymbol{k}) \frac{\mathrm{d} k^{\mu}}{\mathrm{d} \ell} \frac{\mathrm{d} k^{\nu}}{\mathrm{d} \ell}},
$$

then the local flatness condition $g_{\mu \nu}(\boldsymbol{k}) \propto \delta_{\mu \nu}$ implies that $d\left(\boldsymbol{k}_{1}, \boldsymbol{k}_{2}\right)$ is a function of $\left|\boldsymbol{k}_{1}-\boldsymbol{k}_{2}\right|$ only. The condition of local flatness on the Fubini-Study metric tensor has two important consequences. First, the one-body dispersion generated by the particle-hole transformation on the translational invariant two-body interaction is a constant according to Eq. (5.3b), as is the case for the Landau levels in the FQHE. Second, as shown by Roy in Ref. 43, the algebra of projected density operators closes, as is the case for the Landau levels in the FQHE. Thus, it is the departure from a flat (Fubini-Study) metric tensor that can endow the stability of a FCI with a subtle dependence on a nonvanishing bandwidth, as we exemplify next.

We now return to the numerical study of the model defined in Eq. (2.7), in order to explore the effect of a band dispersion quantitatively. To do so, we need a measure for the stability of a phase that we identify by a set of quasidegenerate ground states of small lattices.

One measure for the stability of the phase is simply the size of the gap $\Delta$ above the ground-state manifold, i.e., the difference in energy between the highest of a tuple of quasidegenerate ground-state energies and the lowest-energy eigenvalue above it.

A second measure for the stability of the phase is the correlation length $\xi$ characterizing the exponential decay of the ground-state expectation values of products of a pair of local operators separated by the distance $\boldsymbol{r}$. The shorter $\xi$ is, the farther is a ground state from a quantum phase transition. If a manifold of ground states consists of topologically degenerate states as is the case for the FCI, they become exactly degenerate in the thermodynamic limit with the property that no local operator can transform one of the ground states into another. For a finite system, in contrast, a splitting $\delta$ in energy between the highest and the lowest of the quasidegenerate ground states is to be generically expected. This splitting can be used as a 
(a)

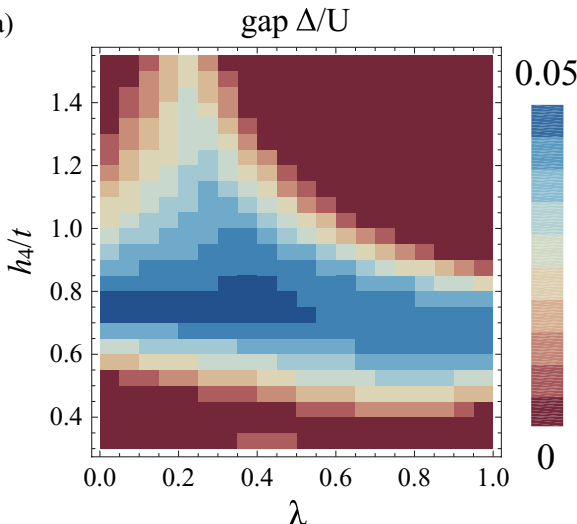

(b)

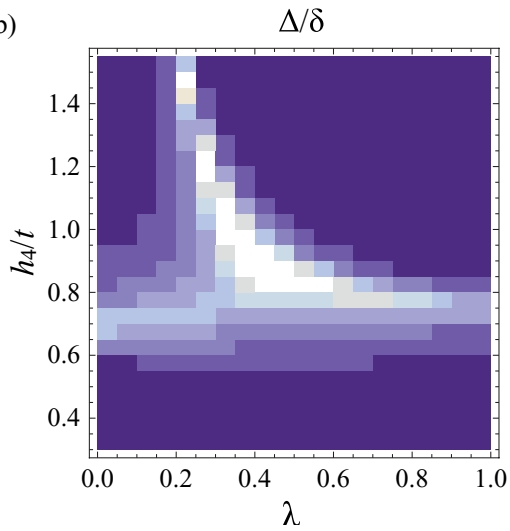

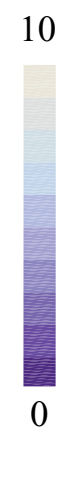

(c) spectrum at $h_{4}=1.05$

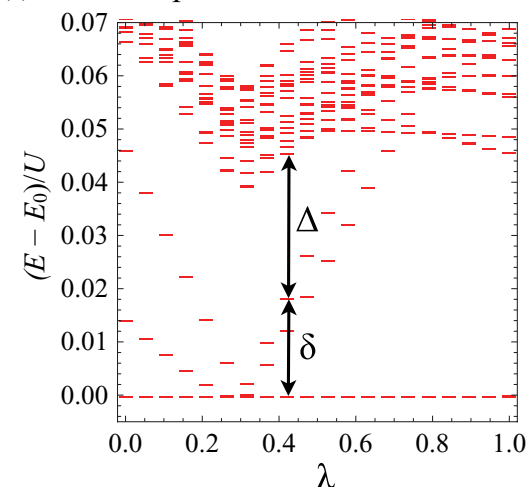

FIG. 6. (Color online) Stability of the FCI phase at $v=1 / 5$ for $C=2$ as the bandwidth of the noninteracting Hamiltonian is changed via the parameter $\lambda$ defined in Eq. (2.7). Panels (a) and (b) show the gap $\Delta$ between the fifth- and sixth-lowest energy eigenvalue of the many-body Hamiltonian and the quotient $\Delta / \delta$, where $\delta$ is the spread in energy of the five lowest eigenvalues, respectively. The region colored blue in (a) and bright (b) is interpreted as a FCI with $v=1 / 5$. In panel (c) the evolution of the lower portion of the many-body spectrum with increasing $\lambda$ at constant $h_{4}=1.05 t$ is plotted. For all figures the number of particles is $N=6$ particles, while $L_{1}=6, L_{2}=5, h_{i}=0, i=1,2,3$, and $U=V=1.5 t$.

measure of $\xi$ through the ansatz

$$
\frac{\Delta}{\delta} \propto e^{L / \xi}
$$

where $L$ is the characteristic linear size of the system.

For concreteness, we focus on the FCI at $v=1 / 5$ with a fivefold quasidegenerate ground state. We interpolate between the flat band and the original noninteracting Hamiltonian with the help of the family of noninteracting Hamiltonians (2.7) parametrized by $\lambda \in[0,1]$ and calculate how the stability of the candidate FCI phase changes, as measured by $\Delta$ and $\Delta / \delta$. Varying $\lambda$ from 0 to 1 makes it possible to change the bandwidth $W$ of the lower band relative to the energy scale of the interaction. The bandwidth of the Bloch band is thus given by $W=\lambda W_{0}$, where $W_{0}$ is the bandwidth of the noninteracting band, that depends on the parameters of the model, $h_{\mu}$ in our case. We do, however, project the Hilbert space to the one spanned by the single-particle states of the lower band for all values of $\lambda$.

We present in Fig. 6 both $\Delta$ and $\Delta / \delta$ as a function of the bandwidth-parameter $\lambda$ and the parameter $h_{4}$ of the noninteracting Hamiltonian (2.1)), where the bandwidth is $W=\lambda \max \left\{\sqrt{2},\left|\sqrt{2}-h_{4}\right|\right\}$. While the largest absolute gap $\Delta$ is indeed obtained for the limit of flat bands $\lambda=0$ in this parameter space, we observe that for a large range of values of $h_{4}$ both the gap $\Delta$ as well as the quotient $\Delta / \delta$ can be substantially increased as $\lambda$ becomes larger. We interpret this as an increase in the stability of the FCI phase with increasing bandwidth of the noninteracting band and exemplify this scenario for $h_{4}=1.05 t$ in Fig. 6(c). Furthermore, we observe that the FCI is stable against a substantial bandwidth of the order of the interaction energy scale for $h_{4} \approx 0.7 t$.

\section{CONCLUSIONS}

The search of materials that realize FCIs requires the hierarchy of energy scales

$$
W \ll V \ll m,
$$

with the largest and smallest energy scale the band gap $m$ and the bandwidth $W$ of the noninteracting band structure, respectively, while the intermediary energy scale $V$ is the characteristic interaction energy. This work assumes the limit

$$
W / m \ll V / m \rightarrow 0
$$

and deals with the energetic question of what is the optimum ratio of $W / V$ needed to stabilize FCIs.

In this work we have established that fractional Chern insulator phases can be stabilized in situations that differ considerably from the ordinary Landau level paradigm, namely a flat band with Chern number 1 . We have characterized the phase diagram of a model that hosts Bloch bands with Chern number either one or two by means of exact diagonalization. Together with the usual characterizations via flux insertion and the counting of degenerate ground states, we have mapped out the real space density profile of several phases that appear in this model at filling $v=1 / 5$ for $C=2$ : CDW, phase separation, and fractional Chern insulator. We showed that the gaps for the $v=1 / 5$ fractional Chern insulating state with $C=2$ are more than one order of magnitude larger than those for the most stable state that we found in the model for $C=1$, at $v=1 / 3$. From these studies we conclude that the most favorable conditions to stabilize a FCI need not be those with $C=1$.

By analyzing the phase boundaries of the $v=1 / 5$ fractional Chern insulating state for $C=2$, we have found instances for which the flat band condition is not optimal. Instead, a nonvanishing bandwidth is. In these examples, the stability of the FCI first increases with bandwidth, reaches a maximum, and then decreases again as too large a bandwidth compared to the interaction energy scale disfavors the topological state. We have thus shown that it is possible to enhance the stability of a fractional Chern insulator by moving away from the condition of flat bands.

The energetic question of when electron-electron interactions can select strongly correlated phases of matter supporting topological order is much more subtle when electrons populate 
the Bloch levels of a Chern band insulator instead of the lowest Landau level. This difference manifests itself with the fact that the Berry curvature is never constant throughout the BZ in the former case, whereas it is perfectly flat for the lowest Landau level in the latter case. In this sense, the competition between the kinetic energy and the electron-electron interaction is much richer for FCIs than it is for the FQHE from the lowest Landau level. These subtleties highlight the importance of the interplay between topology and energetics.

\section{ACKNOWLEDGMENTS}

We thank A. Bernevig and R. Thomale for insightful discussions. This work was supported in part by DOE Grant No. DEFG02-06ER46316 and by the Swiss National Science Foundation. A.G.G. acknowledges conversations with M.A.H. Vozmediano and support from Spanish Grants No. FIS2008-00124, No. FIS2011-23713, No. and PIB2010BZ00512 (Brazil).
${ }^{1}$ D. C. Tsui, H. L. Stormer, and A. C. Gossard, Phys. Rev. Lett. 48, 1559 (1982).

${ }^{2}$ R. E. Prange and S. M. Girvin (eds.), The Quantum Hall Effect (Springer, New York, 1987).

${ }^{3}$ Xiao-Gang Wen, Quantum Field Theory of Many-Body Systems (Oxford University Press, New York, 2004).

${ }^{4}$ T. Neupert, L. Santos, C. Chamon, and C. Mudry, Phys. Rev. Lett. 106, 236804 (2011).

${ }^{5}$ D. N. Sheng, Z. Gu, K. Sun, and L. Sheng, Nat. Commun. 2, 389 (2011).

${ }^{6}$ Y.-F. Wang, Z.-C. Gu, C.-D. Gong, and D. N. Sheng, Phys. Rev. Lett. 107, 146803 (2011).

${ }^{7}$ N. Regnault and B. A. Bernevig, Phys. Rev. X 1, 021014 (2011).

${ }^{8}$ B. A. Bernevig and N. Regnault, Phys. Rev. B 85, 075128 (2012).

${ }^{9}$ Y.-F. Wang, H. Yao, Z.-C. Gu, C.-D. Gong, and D. N. Sheng, Phys. Rev. Lett. 108, 126805 (2012).

${ }^{10}$ Y.-L. Wu, B. A. Bernevig, and N. Regnault, Phys. Rev. B 85, 075116 (2012).

${ }^{11}$ T. Liu, C. Repellin, B. A. Bernevig, and N. Regnault, arXiv:1206.2626.

${ }^{12}$ Y.-F. Wang, H. Yao, C.-D. Gong, and D. N. Sheng, Phys. Rev. B 86, 201101(R) (2012).

${ }^{13}$ Z. Liu, E. J. Bergholtz, H. Fan, and A. M. Läuchli, Phys. Rev. Lett. 109, 186805 (2012).

${ }^{14}$ A. M. Läuchli, Z. Liu, E. J. Bergholtz, and R. Moessner, arXiv:1207.6094.

${ }^{15}$ Z. Liu and E. J. Bergholtz, arXiv:1209.5310.

${ }^{16}$ B. A. Bernevig (private communication).

${ }^{17}$ S. A. Parameswaran, R. Roy, and S. L. Sondhi, Phys. Rev. B 85, 241308(R) (2012).

${ }^{18}$ R. Shankar and G. Murthy, arXiv:1108.5501.

${ }^{19}$ T. Neupert, L. Santos, S. Ryu, C. Chamon, and C. Mudry, Phys. Rev. B 86, 035125 (2012).

${ }^{20}$ B. Estienne, N. Regnault, and B. A. Bernevig, arXiv:1202.5543.

${ }^{21}$ G. Murthy and R. Shankar, arXiv:1207.2133.

${ }^{22}$ The importance of one-body terms was understood during a discussion between Neupert and Bernevig in 2011 to resolve the apparent paradox that Ref. 23 was finding numerical evidences for a FCI at the filling fraction $2 / 3$ while Bernevig and Regnault in Ref. 24 were not. In effect, Ref. 23 studies $\widetilde{H}$ while Ref. 24 studies $H$ at the filling fraction $2 / 3$.

${ }^{23}$ T. Neupert, L. Santos, S. Ryu, C. Chamon, and C. Mudry, Phys. Rev. B 84, 165107 (2011).

${ }^{24}$ B. A. Bernevig, Inversion Symmetric Topological Insulators and Fractional Chern Insulator, talk given at the Kavli Institute for Theoretical Physics China at the Chinese Academy of Sciences, 29 August 2011.

${ }^{25}$ N. Read, Phys. Rev. Lett. 65, 1502 (1990).

${ }^{26}$ B. Blok and X. G. Wen, Phys. Rev. B 42, 8133 (1990).

${ }^{27}$ B. Blok and X. G. Wen, Phys. Rev. B 43, 8337 (1991).

${ }^{28}$ X. G. Wen and A. Zee, Phys. Rev. B 46, 2290 (1992).

${ }^{29}$ X. G. Wen and A. Zee, Phys. Rev. Lett. 69, 1811 (1992).

${ }^{30}$ A. Kol and N. Read, Phys. Rev. B 48, 8890 (1993).

${ }^{31}$ T. Neupert, L. Santos, C. Chamon, and C. Mudry, Phys. Rev. B 86, 165133 (2012).

${ }^{32}$ D. J. Thouless, Phys. Rev. B 40, 12034 (1989).

${ }^{33}$ The transformation law obeyed by $\mathcal{O}(\boldsymbol{r})$ under the translation $\boldsymbol{r}=\boldsymbol{r}^{\prime}+\left(\boldsymbol{r}-\boldsymbol{r}^{\prime}\right)$ is $\mathcal{O}(\boldsymbol{r})=U_{\boldsymbol{r}-\boldsymbol{r}^{\prime}}^{\dagger} \mathcal{O}\left(\boldsymbol{r}^{\prime}\right) U_{\boldsymbol{r}-\boldsymbol{r}^{\prime}}$, with the unitary matrix $U_{\boldsymbol{r}}:=\operatorname{diag}\left(e^{\mathrm{i} r \cdot \boldsymbol{Q}_{1}}, \ldots, e^{\mathrm{i} \boldsymbol{r} \cdot \boldsymbol{Q}_{n}}\right)$ that represents the action of the translation operator in the basis that we chose for the ground-state manifold.

${ }^{34}$ R. Tao and D. J. Thouless, Phys. Rev. B 28, 1142 (1983).

${ }^{35}$ E. H. Rezayi and F. D. M. Haldane, Phys. Rev. B 50, 17199 (1994).

${ }^{36}$ E. J. Bergholtz and A. Karlhede, Phys. Rev. Lett. 94, 026802 (2005); J. Stat. Mech.: Theory Exp. (2006) L04001.

${ }^{37}$ E. J. Bergholtz and A. Karlhede, Phys. Rev. B 77, 155308 (2008).

${ }^{38}$ B. A. Bernevig and N. Regnault, arXiv:1204.5682.

${ }^{39}$ B. S. Shastry and B. Sutherland, Phys. Rev. Lett. 65, 243 (1990).

${ }^{40}$ D. N. Sheng, L. Balents, and Z. Wang, Phys. Rev. Lett. 91, 116802 (2003).

${ }^{41}$ R. G. Melko, A. Paramekanti, A. A. Burkov, A. Vishwanath, D. N. Sheng, and L. Balents, Phys. Rev. Lett. 95, 127207 (2005).

${ }^{42}$ D. N. Page, Phys. Rev. A 36, 3479 (1987).

${ }^{43}$ R. Roy, arXiv:1208.2055. 